\title{
A type description language supporting interoperability in open distributed systems
}

\author{
Wayne Brookes \\ CRC for Distributed Systems Technology, Department of Computer \\ Science, The University of Queensland, Brisbane Qld 4072, Australia. \\ Tel. +6173365 3168. Fax. +61733651999. \\ email:W.Brookes@cs.uq.edu.au
}

\begin{abstract}
A number of existing Interface Definition Languages (IDLs) exist for describing interoperating objects in an open distributed processing (ODP) environment. However these existing languages are limited in their expressive power, and cannot fully describe all the concepts encountered in open distributed systems. This paper proposes a richer type description language that is able to describe objects, their interfaces, interactions and bindings. The language is based on a type model developed for ODP systems and an overview of this type model is presented. The language is described first by example, then more formally using EBNF notation.
\end{abstract}

\section{Keywords}

Distributed systems, type systems, interface definition languages, behaviour specification

\section{INTRODUCTION}

With increasing use of global computer networks, there is a need for an organised form of cooperation between systems. The Reference Model of Open Distributed Processing (RM-ODP) defines a framework for co-operation between object-based systems [8].

One component of this framework is a type model. The type model defines the basic set of types important for interoperability, and provides support for defining a common understanding of the types of data and interactions in an open distributed system. A Type Repository Function is also defined which addresses the use of types in ODP systems. In particular it defines the storage, retrieval and use of types in type matching.

The work reported in this paper has been funded in part by the Cooperative Research Centres Program through the Department of the Prime Minister and Cabinet of the Commonwealth Government of Australia. It was also partially supported by an Australian Government Postgraduate Research Scholarship (APRA). 
There are a number of existing platforms for distributed computing which define type models and type management for use in open distributed systems. These include OSF/DCE [12, 13], OMG/CORBA [11], ANSAware [2], COMANDOS [5] and Concert/C [3]. Further, there are type models developed in related fields which are candidates for use in ODP systems, including ASN.1 [6], GMDO [7] and work on behavioural subtyping by America [1] and Liskov \& Wing [10] .

However an analysis of each of these approaches shows that they have limited type models which do not describe all concepts from ODP systems (e.g. binding) and in many cases, they are for use in homogeneous systems (where all interacting entities are using the same type model). Moreover, all of the platforms for distributed computing have type models which are based on syntax only, whereas the work on behavioural subtyping shows that including elements of behaviour description in type descriptions can improve the accuracy of type matching and substitution [10]. To overcome some of these limitations, at the DSTC we have developed an extended type model which supports additional types not found in many other approaches, and our model also incorporates some elements of behaviour description [4].

For a type model to be useful in a practical environment, it must have an associated language. Many languages are possible for a single type model, differing in their coverage of the model, and syntax. This paper presents a language defined for our type model as presented in [4]. A new language was introduced as existing languages are often highly specific to their underlying type model, making it difficult to extend them. However where possible, features from existing languages have been considered (e.g. basic datatypes) to avoid creating a completely new language.

The purpose of this language is to allow the definition of types for interoperability, including interfaces, interactions, objects, bindings, etc. It differs from existing languages by allowing a broader definition of types, as it is based on a more general type model. However types defined in other models/languages can be mapped into this language. In terms of the Reference Model for Open Distributed Processing, this paper presents a language for describing ODP concepts at the computational level.

The remainder of this paper shows various aspects of the language. Section 2 highlights some of the important features of the type model. Section 3 discusses the requirements for this language, while section 4 presents the language itself. Section 5 gives an example of possible uses of the language, and section 6 concludes the paper. Appendix A shows an EBNF description of the language components described in this paper.

\section{TYPE MODEL}

Underlying any language for describing types there is a type model. This model defines the boundaries of what the language can describe. For supporting interoperability in open distributed systems, the type model must be able to describe interacting objects in distributed systems, including the types of objects, interfaces, interactions and bindings. A number of different approaches exist which are able to describe these concepts to differing degrees $[2,3,5,11,12,13]$.

The type language presented in this paper is based on our type model presented in [4], which is designed as a canonical type model for describing types in open distributed systems. As well as traditional data types and interface types provided by type models of existing IDLs, this model allows the description of the types of interactions between objects, and types of bindings 
between objects. It supports different interaction protocols. It can describe types of relationships between entities in an open distributed system and use meta-types for classification of entities.

Interaction types are a generalisation of the ways in which objects can interact. Other type models support either operations (remote procedure calls), notifications (one-way announcements), streams (continuous data flows) or combinations of these, but all are treated distinctly. Interaction types were created to provide a common way to describe these concepts, as well as less common patterns of interactions (e.g. remote procedure calls which result in a callback).

Each interaction type has a protocol type associated with it. This is how the distinction between kinds of interactions is made. For example, an interaction's protocol type may be remote procedure call, notification, stream or another pattern of interaction. It is important to realise that these are very high-level protocols, and are completely independent of the transport protocol used to actually carry out the interaction. Describing high-level protocol types as separate entities allows new interaction protocols to be introduced into the system, and allows for more flexible matching when the protocols of interacting objects do not match exactly (e.g. one object uses remote procedure call, but another uses message passing).

Another feature of this type model is the ability to describe complex binding types. Many existing type models assume a client-server pattern of interaction, however this is a limited view of interaction patterns in an open distributed system. The binding types described here are capable of supporting multi-party bindings, and any combination of interaction patterns between those parties. This can be used to describe object configurations such as video conferencing, multicast interactions, replicated services, and transformation objects which can intercept and transform interactions.

Relationships exist between many entities in an ODP system, and these relationships need to be described and stored. Other approaches have been to maintain a separate "relationships service" $[8,11]$, however in the model presented here, types of relationships are included as part of the type model. This allows more uniform treatment of relationships, as well as making it easy to maintain repositories of relationship descriptions which can be searched and related to each other (relationships between relationships). From the type model point of view, relationships between types are very important, notably subtyping and substitutability relationships which can be used for matching compatible objects in an open distributed system.

The model also includes facilities for describing meta-types, which are "type of types". Another view is that they are types used for classification purposes. This allows description of types which have no actual physical implementation, but aid in the searching process (e.g. a meta-type "File Server" which is used to group different types of file servers under a common heading). Template types are also supported by the model in order to describe the instantiation of objects in a running system.

\section{LANGUAGE REQUIREMENTS}

Requirements for a type description language can be divided into two broad categories: requirements from the type model, and requirements that come from language design issues. The majority of the interesting requirements presented here are derived from the type model.

The first requirement is the ability to describe the behaviour of action types (interactions, interfaces and bindings). Behaviour description is important in open distributed systems because currently behaviour and semantics is often embedded in the names used for interfaces and 
interactions, and the semantics is implied by having a common understanding of the words used. In an open system, this common understanding cannot always be guaranteed. While there will always be a problem with different interpretations of names used, providing more complete behaviour description can help reduce possible misinterpretations. However it is important to keep in mind that in the context of a language, behaviour description must remain simple. If not, it will not be used by programmers who use the language, and the value of behaviour description is lost. There is a trade-off between the expressive power of the behaviour descriptions and the simplicity of writing them.

A second requirement is that the language should be higher order as it is necessary to pass type information between systems. It is necessary to pass type information to enable dynamic type checking, when all type information may not be found in one location (e.g. a distributed type repository). It is also necessary to pass around "function pointers", which may be "invoked" by the recipient of such a pointer. These will differ from the centralised version of function pointers however, as it is also necessary to record the object to which the pointer belongs, and how it may be accessed.

The language should be as expressive as existing languages. Core concepts for ODP systems that can be described in existing languages should be describable in the new language. Mappings can then be defined between existing languages and the new language. It is recognized that this mapping may be "lossy", i.e. it is possible that not all concepts are able to be mapped.

To satisfy end-users, the language should be simple and easy to understand. At the other end of the spectrum, it should be expressive enough to describe all concepts necessary for supporting interoperability in open distributed systems. These conflicting goals can be partially addressed by providing a descriptive core language, but providing macros to make it simple to write type descriptions for common scenarios (e.g. client-server remote procedure calls).

The language should be object-based, to reflect the object-based nature of ODP systems. It should support inheritance of type descriptions to support code re-use as much as possible. Inheritance of type descriptions can also be used in some cases to automatically determine subtyping relationships.

\section{TYPE LANGUAGE}

This section presents the type language itself. The language is based closely on the type model outlined in the previous section. The language is presented here in the form of a language grammar, shown in Extended Backus-Naur Form (EBNF). Textual descriptions of the EBNF notations are also given, as well as examples of the language elements. A full description of the language concepts discussed in this paper is presented in Appendix A.

Taking a top-down view, the language as a whole consists of a sequence of language elements, and each of these language elements may be a type definition. This paper shows the definition of interaction types, interface types, protocol types and binding types, although the full range of the type model presented earlier is to be described in the language. 


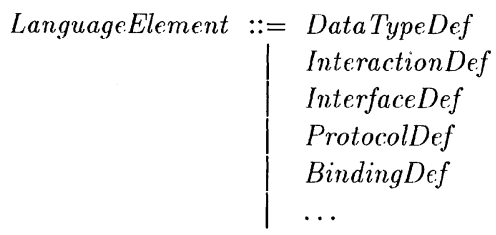

The remainder of this section contains the body of the language, taking the InteractionDef, InterfaceDef, ProtocolDef and BindingDef language elements and expanding on them. Other elements of the type langauge are not shown here.

\subsection{Interaction Types}

Interface types are constructed from interaction types. Consequently, interaction types are commonly defined at the same time as defining an interface. In our model, however, interaction types are first class entities and may appear either inside interfaces, or may be defined external to any interface (globally). Figure 1 shows an example of a globally defined interaction type definition.



Figure 1 An example interaction type definition.

This interaction type is a standard remote procedure call; in this example, to request a file from a file server. The string "RPC.client" defines the protocol that is used by this interaction (RPC protocol, client side). The interaction name is "get" and the client provides a filename as an argument to the interaction in parentheses. The client is prepared to accept three possible terminations: "OK", "noSuchFile" or "permissionDenied". In this example, none of these terminations have any arguments associated with them, although they may. What follows inside brackets ("[", "]") is the description of the interaction behaviour. In this example it is not shown, but may be a description in any suitable behaviour description language (e.g. Liskov \& Wing [10], Basic LOTOS [9]).

The core of the language specification for globally defined interaction types is shown below.

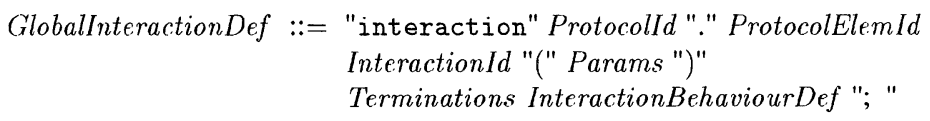

As demonstrated in the example, interaction types consist of the word "interaction" followed by the interaction protocol (ProtocolId.ProtocolElemId), the interaction identifier, parameters in parentheses, followed by terminations and behaviour description. Each of these elements is described in Appendix A. 


\subsection{Interface Types}

As with other approaches $[2,5,11,12]$, our language has interface types as a core component. Interface types describe the visible behaviour of an object, i.e. how an object is capable of interacting with other objects in the system.

Before presenting the language definition for an interface type, an example of this language is shown in Figure 2.

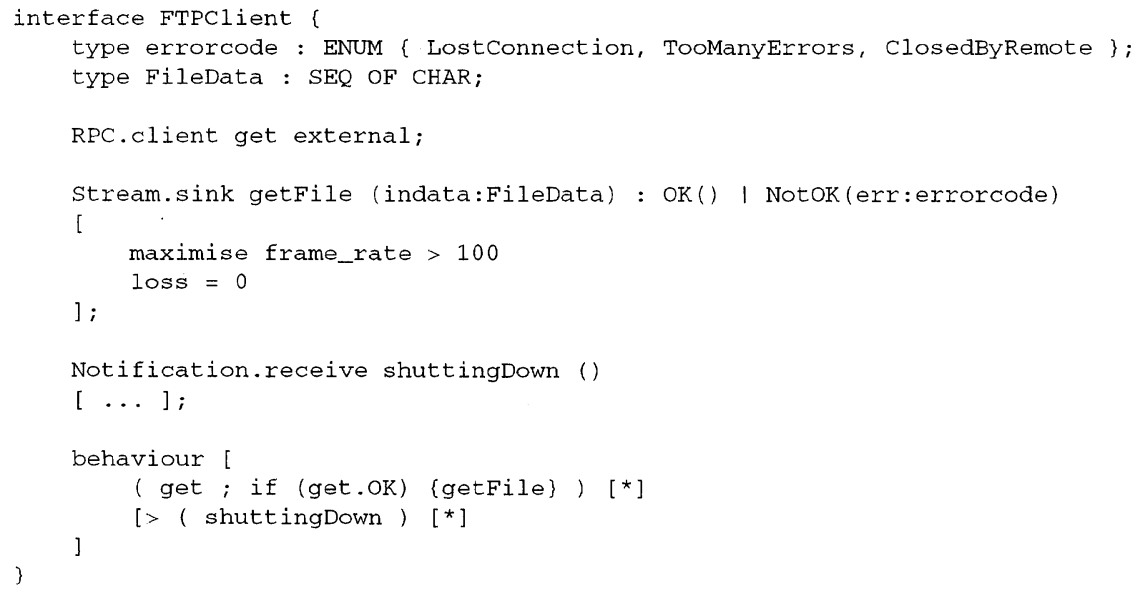

Figure 2 An example interface type definition.

The example is of a fictitious FTP client. This client is limited to retrieving files from an FTP server ("get" operation). The client sends a request to the server to retrieve a file, and the server sends a response, either "OK", or an error indicating why it cannot send the file. This uses a remote procedure call interaction. If the server returned "OK", then the client expects to receive a stream of data, which is the file it requested. At any time, the server may send a notification to indicate that it is about to shut down.

Looking at the syntax of the example, it shows the important components of any interface definition. It contains two data type definitions, several interaction type definitions and a description of the interface behaviour. The first data type defines the possible error codes that may be returned if the stream interaction terminates abnormally, while the second defines the type of data sent through the stream.

The first interaction type is the "get" operation defined earlier (globally). The type declaration simply states that the interaction uses the "RPC.client" protocol, the interaction name "get", and is defined externally to this interface.

The second interaction type is the stream data which contains the file being received. Again it has a protocol, "Stream.sink" (Stream protocol, sink side) and an interaction name, "getFile". The argument to the interaction is the type of data which will be flowing through the stream. The stream has two terminations, "OK", and "NotOK", and the "NotOK" termination has an argument 
associated with it ("errorcode"). The behaviour description is shown for this interaction, in the form of Quality of Service attributes. In this example, each attribute has a matching indicator ("maximise" or "minimise"), the attribute name, operator and attribute value.

The third interaction type is the notification that the server is shutting down. It has the protocol "Notification.receive" (Notification protocol, receiver side), interaction name "shuttingDown", and no arguments (by definition notifications are one-way and thus have no terminations). Again the behaviour description is not shown in this example, but may be described in any suitable behaviour description language.

The final portion of the example is the description of interface behaviour. This is distinct to the description of behaviour for each interaction, as each interaction can only describe its own behaviour in isolation. When describing behaviour at the interface level, it is possible to describe how the interactions relate to each other. The behaviour description technique here is based on the operators of Basic LOTOS [9] but has been extended to describe the concepts necessary for interface behaviour description.

The behaviour description in the example can be divided into two clauses. These clauses are separated by the interruption operator (" [ $>$ "). The first clause reads as follows: "A get interaction occurs. If the termination of 'get' is 'OK', a getFile interaction occurs (it is implied that if the termination is not ' $\mathrm{OK}$ ', then no interaction occurs)". The second clause simply says "A shuttingDown interaction occurs". The second clause interrupts the first, and the shuttingDown interaction may occur at any time. At the end of each clause is a statement of concurrency, shown in brackets ("[", "]"). In this example, the symbol "*" is used, which represents infinity. This says that an infinite number of occurrences of each clause may occur in parallel with each other. That is, a client may get any number of files simultaneously.

The example interface description shows the basic features of the proposed language for interface types. The interface clause of the language is defined below. Selected pieces are shown here, while the currently defined scope of interface types is shown in Appendix A.

$$
\begin{aligned}
\text { InterfaceDef }::= & \text { "interface" InterfaceId InheritanceDef } \\
& \text { \{"InterfaceBody InterfaceBehaviourDef } "\} "
\end{aligned}
$$

An interface definition consists of the word "interface" followed by the interface identifier, plus a possible definition of inheritance. The interface body and interface behaviour definition follow this in braces (" $\{$ ", " $\}$ ").

$$
\begin{aligned}
\text { InterfaceBody }::=\varnothing & \varnothing \begin{array}{l}
\text { DataTypeDef InterfaceBody } \\
\text { InteractionDef InterfaceBody }
\end{array}
\end{aligned}
$$

The body of an interface may be empty (the null interface, useful for creating inheritance hierarchies), or may contain a sequence of data type definitions and interaction definitions (in no particular order). Data type definitions are not considered further in this paper.

$$
\begin{aligned}
& \text { InteractionBehaviourDef }::=\varnothing \\
& \mid \begin{array}{l}
~[" \text { AttributeDefs "]" } \\
\ldots
\end{array}
\end{aligned}
$$


This paper shows only a limited form of behaviour description within interactions. As shown in the example of a stream protocol earlier, this behaviour is described by a set of attribute definitions.

There is nothing novel in the use of attributes for describing quality of service. The attributes described here allow the definition of ranges of values, and include a "matching hint" to assist with type matching. The matching hint is either unspecified (empty), "minimise" or "maximise". The matching hint is used when performing type matching as done by a trader during service selection or a binder (distributed system infrastructure component) during dynamic binding. An example of the use of a matching hint would be when a service will accept variable qualities of service (a range). Some qualities of service are preferably maximised (e.g. throughput), while many are preferably minimised (delay, jitter, etc.). This paper only presents a simple example of how quality of service attributes may be described, although richer description techniques are possible.

The interface behaviour definition consists of the keyword "behaviour" followed by the behaviour definition elements.

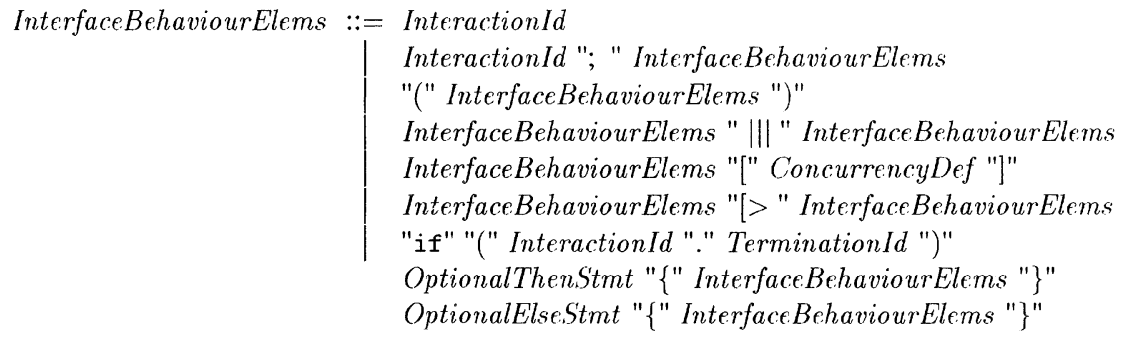

The behaviour definition is trying to capture the legal sequences of interactions that may occur. Each InterfaceBehaviourElem captures one clause of the behaviour specification. As defined above, it may be a single interaction identifier or a sequence of interaction identifiers separated by semi-colons. A clause may be scoped by enclosing it in parentheses. Clauses may occur in parallel, separated by the parallelism operator $(|| \mid)$. A clause may be followed by a concurrency definition indicating how many occurrences of that clause may occur in parallel with each other. The final clause form is an if-then-else structure. If a particular termination occurred as a result of the preceding interaction, then one behaviour clause is chosen, else a different clause is chosen.

The behaviour description presented here is modelled on the operators of Basic LOTOS [9]. Elements such as the if-then-else clause have no more expressive power than concepts from Basic LOTOS (e.g. guarded commands with choice), but are syntactically easier to read. There is a direct mapping between an if-then-else clause and the equivalent Basic LOTOS, and such mapping of this and other behaviour description concepts is the subject of ongoing work on the type language.

\subsection{Protocol Types}

A feature unique to this language in the context of ODP systems is the notion of protocol types. Protocol types distinguish the different forms of interaction that are possible, e.g. RPC, Stream, Notification, or more complex patterns of interaction. 
An example of two different protocol types is shown in Figure 3. This example defines two basic protocol types. In an ODP environment, common types like these would be predefined: they are shown here for illustration purposes.



Figure 3 An example protocol type definition.

The first is the remote procedure call (RPC) protocol. It begins with a local data type definition for enumerating the possible RPC semantics at a high level. The following two items define the protocol elements. These define the participants in the protocol. Although these definitions look similar to definitions of procedural operations, they are not operations: they are never "invoked", but are provided for defining the protocol between interacting entities. For remote procedure call, there are two interacting entities, "client" and "server". The items in parentheses define the messages exchanged by this protocol. For RPC, a client element will 
generate a "request" message, and will receive a "response" message. An RPC server element mirrors this, receiving a "request" message and generating a "response" message. The final item in the description of a protocol element is the behaviour specification. In this language, this defines valid sequences of messages that can be exchanged as part of the protocol (although one would expect protocol behaviour to be more complex in general). For both RPC clients and servers, they process a request message first, followed by the response message.

Also included in protocol definitions is a set of attribute types that are related to the protocol. These are attribute types, not instances: the attribute instances occur in interface types which make use of the protocol. Attribute types may be optional, and may have default values. The attribute type shown for RPC, "RPCSemantics" is optional, has a type associated with it, and has a default value.

The second example of a protocol type shown is for a stream protocol. Again it has two interacting entities, "source" and "sink". A stream source generates a sequence of stream packets (the behaviour description contains a "*" which captures the notion of "many" of the specified message occuring), followed by a distinguished end-of-file packet, and a stream termination. The stream protocol has many attributes associated with it, many of these describing quality of service aspects of the protocol.

Highlights of the language definition for protocol types is as follows:

$$
\begin{gathered}
\text { ProtocolDef ::= "protocol" Protocolld InheritanceDef } \\
\text { "\{"ProtocolBody" }\} "
\end{gathered}
$$

A protocol definition consists of the keyword "protocol" followed by the protocol identifier, inheritance specification and the body of the protocol definition.

Each protocol behaviour element may have behaviour associated with it. A full description of possible protocol behaviours is not shown here. In this paper, the description of protocol behaviour is limited to two operators, sequencing and choice.

$$
\begin{aligned}
\text { ProtocolBehaviourElems }::=\text { ProtocolBehaviourElem } \\
\qquad \begin{array}{l}
\text { ProtocolBehaviourElem "; "ProtocolBehaviourElems } \\
\text { ProtocolBehaviourElems "[]" ProtocolBehaviourElems }
\end{array}
\end{aligned}
$$

\subsection{Binding Types}

Crucial to ODP systems is the concept of binding. In many other systems, binding is handled by the infrastructure, but is not described at the language level, or else only selected pieces of binding information are described.

This language takes the approach of describing the types of bindings that are expected to occur. In many ways, this makes binding types a central part of the model, as they describe the configurations of objects (and their interfaces) and communication that can occur between them. An example of a binding type in this language is shown in Figure 4.

This example shows a non-traditional FTP binding. It includes a filter which is applied to the data stream being transmitted. The scenario is that a client requests a file using the "get" RPC operation, and after the RPC returns, is sent the requested file via a stream. The binding described in Figure 4 specifies that the stream will pass through a filter before reaching the original client. 


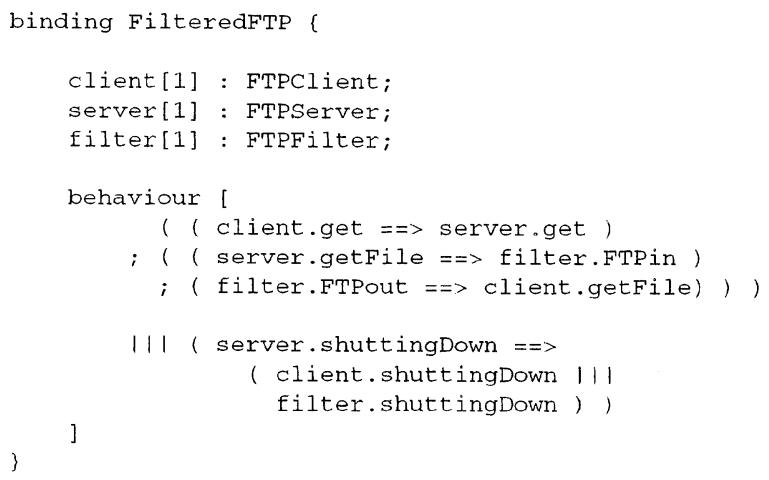

Figure 4 An example binding type definition.

The description is divided into two main parts: role specification and behaviour specification. The first part is the role specification which shows the interface types that participate in the binding, and their cardinalities. In the example shown, the binding consists of one FTPClient interface, one FTPServer interface, and one FTPFilter interface. Only the simple case is shown, however higher cardinalities are permitted.

The second part of the description is the behaviour specification. The behaviour of a binding describes how interactions are passed between participants in the binding - essentially routing information. In this behaviour specification, a causal ordering operator has been introduced $("==>$ ). This operator is used in specifying the causality between specific interactions (message delivery). It was introduced to capture the additional semantics of causality that the LOTOS sequencing operator (";") does not capture.

The behaviour specification in the example shows that the client role participates in a "get" interaction, which is causally followed by the server role participating in a "get" interaction. This is then followed by the server undertaking a "getFile" interaction, causally followed by the filter performing an "FTPin" interaction. Finally the filter participates in an "FTPout" interaction, causally followed by the client participating in a "getFile" interaction.

In parallel with this behaviour is the behaviour of the "shuttingDown" interaction. It acts in a multicast manner, with the interaction "shuttingDown" occuring at the server role, causally followed by the "shuttingDown" interaction occuring at both the filter and the client roles. In the specification of the binding behaviour, the occurrence of a "shuttingDown" interaction does not terminate the binding, which is why it is in parallel with the other interactions in the binding. In this example, destruction of the binding is assumed to be undertaken by a party external to the binding itself (i.e. one of the participant roles, or the infrastructure).

The remainder of this section defines the language for describing binding types.

$$
\begin{aligned}
\text { BindingDef }::= & \text { "binding" BindingId InheritanceDef } \\
& \text { "\{"BindingRoleSpec BindingBehaviourSpec " }\} "
\end{aligned}
$$

A binding type definition consists of the keyword "binding", followed by an optional 
specification of inheritance. The body of the binding type has two components: the role specification and the behaviour specification.

A binding may have many roles. Each role in the binding consists of an interface identifier, an optional specification of cardinality and the interface type of the role.

$$
\text { RoleDef ::= InterfaceId RoleCardinalityDef " : InterfaceTypeId }
$$

In addition to roles, bindings may include a behaviour specification, which consists of binding behaviour elements.

$$
\begin{aligned}
& \text { BindingBehaviourElems ::= QualifiedInteractionId } \\
& \qquad \begin{array}{l}
\text { QualifiedInteractionId "; " BindingBehaviourElems } \\
\text { QualifiedInteractionId "==> "BindingBehaviourElems } \\
\text { BindingBehaviourElems " ||| "BindingBehaviourElems } \\
\text { "(" BindingBehaviourElems ")" }
\end{array}
\end{aligned}
$$

A limited number of binding behaviours have been defined so far. The current list is not exhaustive, but demonstrates the purpose of a binding behaviour description. As defined above, a behaviour description may be a single interaction (qualified by the interface to which the interaction belongs), an interaction followed by some other behaviour, an interaction which causally results in some other behaviour or two behaviour descriptions in parallel. Behaviour descriptions may also be grouped with parentheses.

That concludes the presentation of the type language. Based on the description it can be seen that this language meets the requirements presented earlier, with the focus being on the ability to describe interaction, interface, protocol and binding behaviour.

\section{USAGE SCENARIO}

The previous section presented elements of the language. But a language is only useful when it is shown in the context in which it is to be used. This section discusses possible usage scenarios for the language.

Firstly, statements in the type language can be parsed and checked for consistency errors. This ensures that the language can support static type safety. Once a parsed version of the language exists (e.g. stored as an abstract syntax tree or a syntax graph), it can be used for a number of purposes, including:

- generation of stubs for objects, given a definition of object and interface types,

- automatic generation of binding state information at run-time, possibly including checks that the described binding behaviour is satisfied by the actual binding. This is feasible for simple binding behaviour descriptions (e.g. those that can be mapped to finite state machines), but beyond simple behaviours checking becomes difficult and in certain cases impossible, depending upon the level of detail described in the binding behaviour,

- integration of type information into the software engineering environment, through the use of a type manager and trader to add persistence to type descriptions. This would make type descriptions available to programmers during the software development process (compiling, linking, etc.) 
These are only examples of how the type language shown in this paper could be used in practice. The uses presented above are all feasible (for simple descriptions of binding behaviour), but the implementation of these is left for future work.

\title{
6 CONCLUSION
}

This paper presents a subset of a language for supporting interoperability in open distributed systems. The language differs from similar approaches as it describes a richer set of types, including interaction types, binding types and interaction protocol types. It also provides a richer description of some types by including components of behaviour description.

The paper gave an overview of the underlying type model, highlighting the features which differ from other models. Then the requirements of a language were described, including both requirements which are derived from the model and those which are specific to the language.

Four components of the language were presented: interaction types, interface types, protocol types and binding types. For each of these, an example was shown and described, followed by selected elements of the language described using EBNF syntax. Finally, an example of how this language might be used in practice was given.

There is still much work to do on type languages for open distributed systems. In particular, the correct balance must be found between satisfying the needs of end users (programmers) and the system infrastructure, as each requires a different level of abstraction. Further work is also necessary on behaviour description (including subtyping of behaviour descriptions) to create languages which describe ODP concepts while not producing unwieldy descriptions.

\section{A EBNF DESCRIPTION}

\section{A.1 Overall language}

$$
\begin{aligned}
& \text { Type Language }::=\text { LanguageElement } \\
& \mid \text { LanguageElement TypeLanguage }
\end{aligned}
$$

\section{A.2 Interaction types}

\author{
InteractionDef $::=$ ScopedInteractionDef | GlobalInteractionDef \\ ScopedInteractionDef $::=$ DefinedInteractionDef | ExternalInteractionDef \\ DefinedInteractionDef ::= OptionalInteractionKeyword ProtocolId "." ProtocolElemId \\ InteractionId "(" Params")" \\ Terminations InteractionBehaviourDef ": "
}


ExternalInteractionDef $::=$ OptionallnteractionKeyword ProtocolId "." ProtocolElemId InteractionId "external" "; "

OptionallnteractionKeyword $::=\varnothing \mid$ "interaction"

GlobalInteractionDef ::= "interaction" Protocolld "." ProtocolElemId InteractionId "(" Params ")"

Terminations Interaction BehaviourDef "; "

Params ::=ø

Param

Param ", Params

Param ::= ParamId": " TypeId

Terminations ::= | " " TerminationList

TerminationList $::=$ Termination

Termination " |" TerminationList

Termination $::=$ TerminationId "(" Params ")"

InteractionBehaviourDef $::=\varnothing$

$\mid \begin{aligned} & \text { "[" AttributeDefs "]" } \\ & \text {.. }\end{aligned}$

\section{A.3 Interface types}

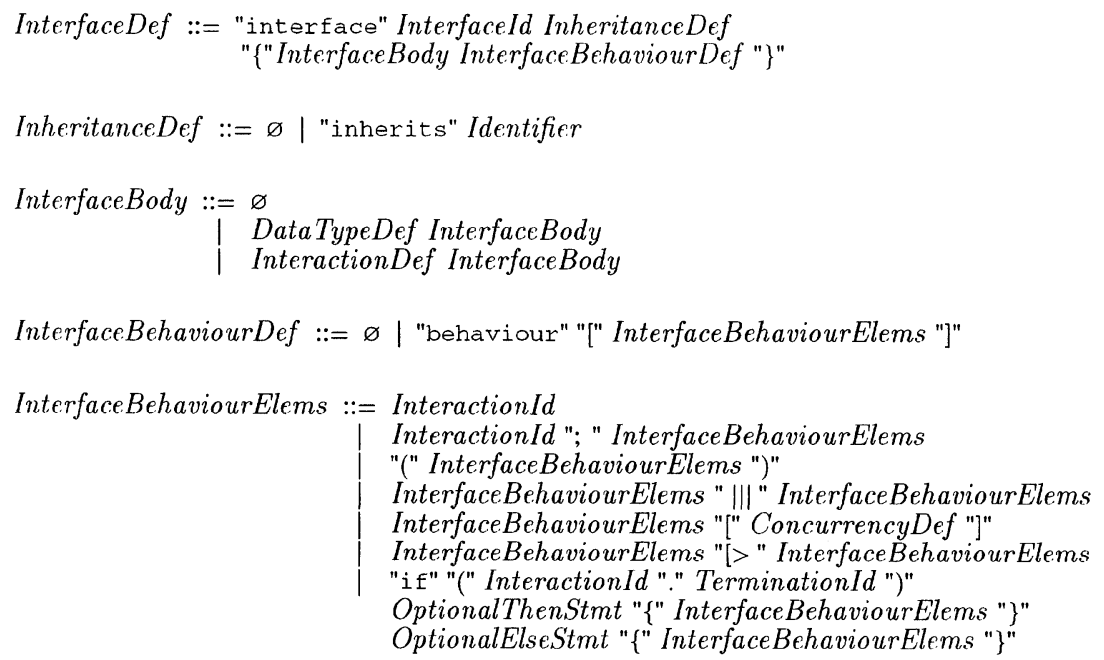




\section{A.4 Protocol types}

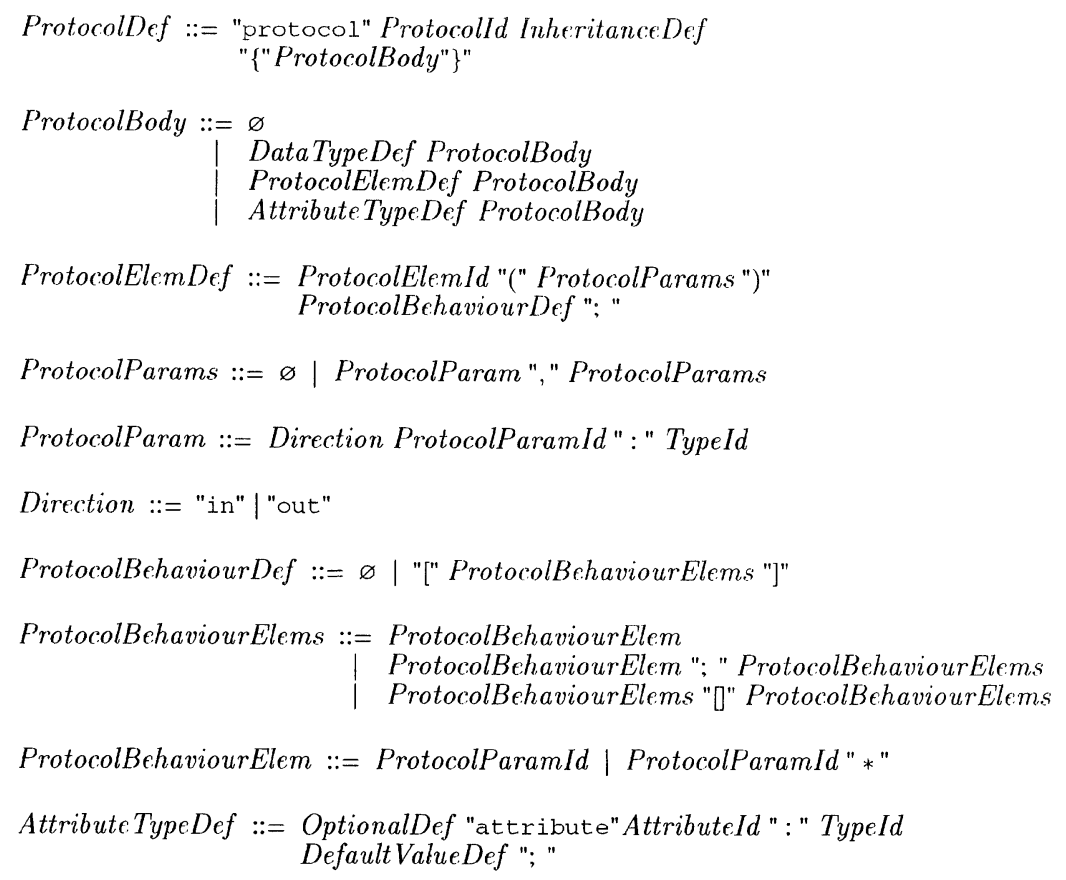

\section{A.5 Binding types}

$\begin{aligned} \text { BindingDef }::= & \text { "binding" BindingId Inheritance Def } \\ & \text { " }\{\text { BindingRoleSpec BindingBehaviourSpec " }\} "\end{aligned}$

BindingRoleSpec ::=ø | RoleDef BindingRoleSpec

RoleDef ::= InterfaceId RoleCardinalityDef " : " InterfaceTypeId

RoleCardinalityDef ::=ø| "["InfiniteNumber"]"

BindingBehaviourSpec ::=ø | "behaviour" "[" BindingBehaviourElems "]"

BindingBehaviourElems ::= QualifiedInteractionId

QualifiedInteractionId "; " BindingBehaviourElems QualifiedInteractionId " ==> " BindingBehaviourElems BindingBehaviourElems " ||| " BindingBehaviourElems | "(" BindingBehaviourElems ")"

QualifiedInteractionId ::= InterfaceId "." InteractionId 


\section{ACKNOWLEDGEMENTS}

I would like to thank my colleagues from the CRC for Distributed Systems Technology (DSTC) for their discussions and helpful comments during the preparation of this paper. In particular, I would like to thank David Arnold, Andrew Berry, Andy Bond, Jadwiga Indulska, Kerry Raymond, Andreas Vogel and other members of the Architecture Unit.

\section{REFERENCES}

[1] P. America. "Designing an Object-Oriented Programming Language with Behavioural Subtyping". In Foundations of Object-Oriented Languages, number 489 in Lecture Notes in Computer Science, pages 60-90, REX School/Workshop, Noordwijkerhout, The Netherlands. Springer-Verlag, 1991.

[2] APM Ltd, Cambridge UK. ANSAware 4.1 Application Programmer's Manual, Mar. 1992. Document RM.102.00.

[3] J. Auerbach, A. Goldberg, G. Goldszmidt, A. Gopal, M. Kennedy, J. Russell, and S. Yemini. "Concert/C Specification: Definition of a Language for Distributed C Programming”. Technical Report RC18994, IBM T. J. Watson Research Center, July 1993.

[4] W. Brookes, A. Berry, A. Bond, J. Indulska, and K. Raymond. "A type model supporting interoperability in open distributed systems". In Proceedings of the First International Conference on Telecommunications Information Networking Architecture, TINA '95, pages 275-289, Feb. 1995.

[5] V. Cahill, R. Balter, N. Harris, and X. Rousset de Pina. The COMANDOS Distributed Application Platform. Volume 1. ESPRIT Research Reports (Project 2071). Springer-Verlag, 1993.

[6] ISO/IEC DIS 8824-1. Information Technology-Open Systems Interconnection-Abstract Syntax Notation One (ASN.1). Part 1: Specification of Basic Notation, 1992.

[7] ISO/IEC IS 10165-4. Information Technology - Open System Interconnection - Structure of Management Information - Part 4: Guidelines for the Definition of Managed Objects, 1992.

[8] ISO/IEC IS 10746-3. International Standard 10746-3, ITU-T Recommendation X.903: Open Distributed Processing - Reference Model — Part 3: Architecture, Jan. 1995.

[9] ISO/IEC IS 8807. LOTOS, A Formal Description Technique based on the Temporal Ordering of Observational Behaviour, 1988.

[10] B. Liskov and J. M. Wing. “A New Definition of the Subtype Relation”. In O. Nierstrasz, editor, Proceedings of the European Conference on Object Oriented Programming, ECOOP '93, number 707 in Lecture Notes in Computer Science, pages 118-141, Springer-Verlag, Kaiserslautern, Germany, July 1993.

[11] Object Management Group and X/Open. The Common Object Request Broker: Architecture and Specification, 1992.

[12] W. Rosenberg and D. Kenney. Understanding DCE. Open System Foundation, 1992.

[13] J. Shirley. Guide to Writing DCE Applications. Open System Foundation, 1992.

\section{BIOGRAPHY}

Wayne Brookes is currently completing his $\mathrm{PhD}$ in the area of open distributed systems at The University of Queensland. His research focus is on using type management techniques to support interoperability in an ODP-like environment, particularly to support resource discovery and system evolution in open distributed systems. He is currently employed by The University of Queensland in a teaching position, and is affiliated with the CRC for Distributed Systems Technology. 